\title{
Research Article \\ TTK, CDC25A, and ESPL1 as Prognostic Biomarkers for Endometrial Cancer
}

\author{
Qiannan Yang, Bojun Yu, and Jing Sun \\ Department of Gynaecology, Shanghai First Maternity and Infant Hospital, Tongji University School of Medicine, \\ Shanghai /201204, China \\ Correspondence should be addressed to Jing Sun; sunjing61867@tongji.edu.cn
}

Received 6 July 2020; Revised 19 October 2020; Accepted 30 October 2020; Published 17 November 2020

Academic Editor: Guangcun Huang

Copyright (C) 2020 Qiannan Yang et al. This is an open access article distributed under the Creative Commons Attribution License, which permits unrestricted use, distribution, and reproduction in any medium, provided the original work is properly cited.

\begin{abstract}
Objective. Endometrial cancer (EC) is one of the most common malignant gynaecological tumours worldwide. This study was aimed at identifying EC prognostic genes and investigating the molecular mechanisms of these genes in EC. Methods. Two mRNA datasets of EC were downloaded from the Gene Expression Omnibus (GEO). The GEO2R tool and Draw Venn Diagram were used to identify differentially expressed genes (DEGs) between normal endometrial tissues and EC tissues. Then, Gene Ontology (GO) and Kyoto Encyclopedia of Genes and Genomes (KEGG) pathway analyses were performed using the Database for Annotation, Visualization and Integrated Discovery (DAVID). Next, the protein-protein interactions (PPIs) of these DEGs were determined by the Search Tool for the Retrieval of Interacting Genes (STRING) tool and Cytoscape with Molecular Complex Detection (MCODE). Furthermore, Kaplan-Meier survival analysis was performed by UALCAN to verify genes associated with significantly poor prognosis. Next, Gene Expression Profiling Interactive Analysis (GEPIA) was used to verify the expression levels of these selected genes. Additionally, a reanalysis of the KEGG pathways was performed to understand the potential biological functions of selected genes. Finally, the associations between these genes and clinical features were analysed based on TCGA cancer genomic datasets for EC. Results. In EC tissues, compared with normal endometrial tissues, 147 of 249 DEGs were upregulated and 102 were downregulated. A total of 64 upregulated genes were assembled into a PPI network. Next, 14 genes were found to be both associated with significantly poor prognosis and highly expressed in EC tissues. Reanalysis of the KEGG pathways found that three of these genes were enriched in the cell cycle pathway. TTK, CDC25A, and ESPL1 showed higher expression in cancers with late stage and higher tumour grade. Conclusion. In summary, through integrated bioinformatics approaches, we found three significant prognostic genes of EC, which might be potential therapeutic targets for EC patients.
\end{abstract}

\section{Introduction}

Endometrial cancer (EC) is the most common gynaecological malignancy, with an increasing incidence in North America, Europe, and more than 20 developed countries elsewhere [1, 2]. There were 61880 new cases and 12160 deaths from EC in the United States in 2019 [3], up from 60050 new cases and 10470 deaths in 2016 [4]. Two histological classifications have been described among endometrioid adenocarcinoma, type I and type II, and seventy to eighty percent of new cases are type I. Type I tumours, which are mostly of lower grade, are mediated by oestrogen and have endometrioid histology. Type II tumours are composed of higher-grade tumours (generally clear cell or serous) and are more common in thinner and older women $[5,6]$. The prognosis of EC patients is related to the stage and grade of the disease. EC patients with stage III or IV endometrial cancer typically have a worse prognosis, and patients with stages I and II have a better survival [7]. Thus, finding more credible prognostic biomarkers is important for improving the therapies for EC, and investigating their role development is important for further elucidation of the underlying mechanisms of EC.

The Gene Expression Omnibus (GEO) database gathers next-generation sequencing data and high-throughput microarrays submitted by the research community constructed and kept by the National Center for Biotechnology 
Information (NCBI). Screening for differentially expressed genes (DEGs), analysis of gene regulatory networks, and exploration of molecular signals and interrelationships can be performed via the GEO database. Thousands of DEGs that may be involved in EC development have been screened from a large number of studies of gene expression profiles of EC in GEO. However, the identification of DEGs in multiple studies has been limited as a result of the different technology platforms used, the different treatment of data across studies, and the heterogeneity of tissues or samples in different independent experiments. We comprehensively analysed microarray data from two gene expression profiling experiments with consideration of the false positive probability of microarray results.

Two initial gene expression profiles (GSE17025 and GSE63678) were downloaded from the GEO database. To identify common DEGs, the GEO2R tool was applied to integrate the expression profiles. Next, Gene Ontology (GO) and Kyoto Encyclopedia of Genes and Genomes (KEGG) pathway analyses were performed using the Database for Annotation, Visualization and Integrated Discovery (DAVID). The Search Tool for the Retrieval of Interacting Genes (STRING) database was then applied to create a protein-protein interaction (PPI) network. Later, the core genes among the DEGs were identified by Cytotype Molecular Complex Detection (MCODE). Additionally, these core DEGs were explored by UALCAN to obtain information on patient prognosis $(P<0.05)$. Moreover, the expression of DEGs between normal endometrial samples and EC samples was validated by Gene Expression Profiling Interactive Analysis (GEPIA) $(P<0.01)$. Then, KEGG pathway enrichment of these designated DEGs was reanalysed by DAVID. TTK, CDC25A, and ESPL1 were found to be significantly enriched in the cell cycle pathway. Last, TTK, CDC25A, and ESPL1 showed higher expression in the late cancer stage and higher tumour grade. In conclusion, TTK, CDC25A, and ESPL1 are associated with poor prognosis in EC and could be potential therapeutic biomarkers that might be beneficial for EC patients.

\section{Materials and Methods}

2.1. Microarray Data Information. GEO (http://www.ncbi .nlm.nih.gov/geo) is an open database of microarray/gene profiles [8]. Two mRNA expression datasets containing microarray data from normal endometrial tissues and EC tissue, GSE63678 [9] and GSE17025 [10], were selected. The GSE63678 microarray data included 5 normal endometrial tissues and 7 EC tissues and were generated using the GPL571 platform Affymetrix Human Genome U133A 2.0 Array. The GSE17025 microarray data included 12 normal endometrial tissues and 91 EC tissues and were generated using the GPL570 platform Affymetrix Human Genome U133 Plus 2.0 Array.

2.2. Identification of DEGs. GEO2R is an interactive web tool that allows users to compare two or more groups of samples in a GEO series to identify genes that are differentially expressed across experimental conditions. The GEO2R online tool (http://www.ncbi.nlm.nih.gov/geo/geo2r/) was applied to recognize DEGs between normal endometrial tissues and EC tissues with an adjusted $P$ value $<0.05$ and $\left|\log _{2} \mathrm{FC}\right|>1$. The Draw Venn Diagram (http:// bioinformatics.psb.ugent.be/webtools/Venn/) can be used to calculate the intersections of the list of elements. Therefore, we used this tool to analyse the raw data in TXT format and obtain the DEGs in the two datasets. Upregulated genes were DEGs with $\log _{2} \mathrm{FC}>1$, while downregulated genes were DEGs with $\log _{2} \mathrm{FC}<-1$.

2.3. Analysis of GO and KEGG Pathway Enrichment. GO is a general annotation method for genes and their RNA or protein products that is used to characterize the biological characteristics of high-throughput genomic data [11]. KEGG is a compendium of databases with information on genomes, chemical materials, biological pathways, diseases, and drugs [12]. As an online bioinformatics tool, DAVID (http:// david.ncifcrf.gov/) is aimed at recognizing the functions of genes or proteins [13]. The DEG enrichment of GO terms (biological process (BP), cell component (CC), and molecular function $(\mathrm{MF})$ ) and KEGG pathways was visualized by DAVID $(P<0.05)[14]$.

2.4. Analysis of PPI Network and Module. As an online tool, STRING (http://string-db.org/) is designed to visualize PPI information [15]. The potential relationships among DEGs were identified by the STRING app in Cytoscape (confidencescore $\geq 0.4$ and maximum number of interactors $=0$ ). Furthermore, modules of the PPI network were validated via the MCODE app in Cytoscape (max.depth $=100$, node score cut - off $=0.2$, degree cut off $=2$, and $k$-score $=2$ ) [16].

2.5. Survival Analysis and $m R N A$ Expression of Core Genes. The expression level of various genes has an impact on patient survival, which can be shown by Kaplan-Meier plots. As a friendly, comprehensive, and interactive web resource for data analysis of cancer omics, UALCAN (http://ualcan .path.uab.edu/index.html) was applied to obtain KaplanMeier plots and gene expression profiles based on TCGA data [17]. The significance of survival impact is measured by the $\log$ rank test $(P<0.05)$. In addition, based on the TCGA and GTEx projects, the level of gene expression in normal endometrial samples and EC samples was validated via GEPIA ( $\left|\log _{2} \mathrm{FC}\right|$ cut-off $=1, P$ value cut-off $=0.01$ ) [18]. Finally, the University of California Santa Cruz (UCSC) Xena website (http://xena.ucsc.edu/), an analytics, visualization, and universal integration tool for analysing and viewing public datasets was used to assess TCGA cancer genomic datasets for EC. We analysed the associations between prognostic genes and clinical features (cancer stage and tumour grade) by $t$ test $(P<0.05)$. The $c$ Bio Cancer Genomics Portal (cBioPortal) is a publicly accessible resource (http://www .cbioportal.org/) $[19,20]$. We downloaded clinical datasets and cancer genomic datasets for EC [21] from the cBioPortal website and analysed the correlations between prognostic gene expression and survival in EC, and the significance of survival impact is measured by the log rank test $(P<0.05)$. 


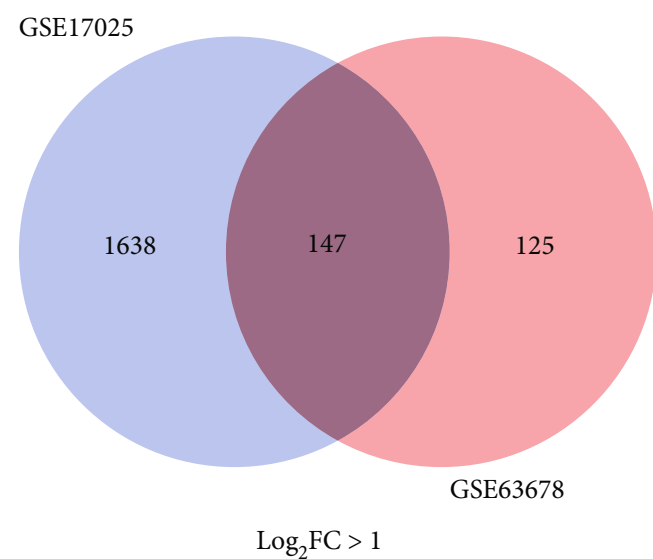

(a)

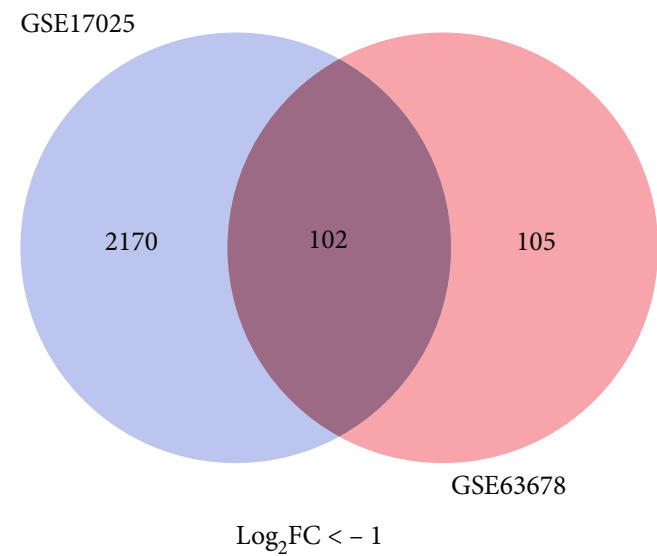

(b)

FIGURE 1: Validation of 249 DEGs in the two datasets (GSE63678 and GSE17025) via Draw Venn Diagram. (a) 147 upregulated DEGs in the two datasets $\left(\log _{2} \mathrm{FC}>1\right)$. (b) 102 downregulated DEGs in two datasets $\left(\log _{2} \mathrm{FC}<-1\right)$.

TABLE 1: All 249 DEGs were detected from two mRNA expression datasets, of which there are 147 upregulated genes and 102 downregulated genes in the EC.

\begin{tabular}{|c|c|}
\hline DEGs & Gene names \\
\hline Upregulated & $\begin{array}{l}\text { ESPL1, E2F8, DHCR24, VAMP8, TSTA3, ANXA1, TPX2, CCNB1, GOT1, ANP32E, LMNB2, KPNA2, PDIA3, BIRC5, } \\
\text { TAGLN2, FOXM1, F2RL1, CDK1, CHEK1, CARMIL1, MUC1, KIF11, GPI, MRPS12, PDXK, EZR, CDC6, HSPA4, } \\
\text { CENPU, PCCB, SMC4, APOBEC3B, AURKA, CLDN7, KIF14, ANXA2P2, MAD2L1, ATP1A1, TJP3, FANCA, BLM, } \\
\text { KIF4A, KRT8, LDHA, SCD, MAP7, MPZL1, UCK2, KIF2C, EIF5A, ANP32A, ACLY, TYMS, MELK, SFN, VDAC1, } \\
\text { CDC20, CENPN, HN1, ZWINT, MPDU1, SAR1A, CCNA2, GTSE1, PBK, TRIP13, S100A11, STAT1, PTTG1, MMP12, } \\
\text { CDC7, CKS2, ISG15, ECT2, KIF23, ANXA2, GSR, TK1, CENPE, ASPM, UBE2S, LMNB1, SPAG5, CDCA3, CKMT1B, } \\
\text { PTBP3, TACC3, UBE2C, CCNB2, PRC1, LRP8, CKAP2, CEP55, PLEKHB2, RRM2, MGST2, CENPA, TOP2A, SYNCRIP, } \\
\text { FEN1, RBM47, MOB1A, KIF18A, KIF15, ST14, SSR1, BUB1B, S100A10, DLGAP5, HJURP, RAD51AP1, ESRP1, ENO1, } \\
\text { MKI67, DTL, GDE1, SULF1, NCALD, ACP1, RAD51, HMMR, TUBB4B, CDCP1, ARF3, KIF20A, MIF, GMDS, SDF2L1, } \\
\text { IDE, CTSZ, DLAT, GAPDH, KIAA0101, MTCH2, TTK, PGD, CDKN3, NME1, NCAPG, MYCBP, BAX, CIT, NEK2, } \\
\text { CENPF, NUSAP1, PGK1, CDC25A }\end{array}$ \\
\hline Downregulated & $\begin{array}{l}\text { H3F3B, HYMAI, NAALAD2, GNAL, MITF, HOXD11, ERG, EVC, HNMT, CA11, GHR, ROR2, KLF3-AS1, BCHE, } \\
\text { SRSF11, WT1-AS, POU6F1, LEFTY2, PHF1, RBPMS, ZNF667, GABBR1, VSTM4, SPAG9, RUNX1T1, PER1, KIAA0368, } \\
\text { PPIEL, TRPC4, SNCA, TSPYL5, ENPP2, UNKL, SOX15, CTSF, NR2F2, ZDHHC17, PNISR, TCEAL2, FOXN3, } \\
\text { KIAA1644, PKD1P1, FBXO9, WNT2, MCOLN3, STAT5B, ENPEP, CBX7, ARMCX1, TBX3, FAM184A, ZFP2, CACNB2, } \\
\text { PEG3, HAND2-AS1, C2orf68, TGFBR3, ZNF37BP, MAF, ZNF135, PGM5, ATRNL1, ST3GAL5, BNC2, AKT3, CYP1B1, } \\
\text { EFS, CDIP1, ZSCAN18, KLF11, ZNF506, MXRA8, UBE2I, TRO, C1orf21, PLAGL1, BHMT2, ST8SIA1, GATAD1, PAK3, } \\
\text { PDS5B, NMT2, CMAHP, MAGEH1, H3F3A, EZH1, CDO1, NUDT11, GSPT2, HNRNPDL, FGF2, CXCL12, IGF1R, } \\
\text { CRBN, GPRASP1, MAGEL2, CHRD, ME3, CIRBP, NUMA1, SNED1, TNS2 }\end{array}$ \\
\hline
\end{tabular}

\section{Results}

3.1. Identification of DEGs in EC. Our study included 17 normal endometrial tissues and 98 EC tissues. A total of 4057 and 479 DEGs were extracted from GSE17025 and GSE63678 using the GEO2R online tool. Then, the DEGs common to the two datasets were identified via the Draw Venn Diagram. Compared with normal endometrial tissues, 249 common DEGs were discovered, of which 147 were upregulated (adjusted $P$ value $<0.05, \log _{2} \mathrm{FC}>1$ ) and 102 were downregulated (adjusted $P$ value $<0.05, \log _{2} \mathrm{FC}<-1$ ) in EC tissues (Figure 1 and Table 1).

3.2. GO and KEGG Pathway Analysis of DEGs in EC. The GO and KEGG pathway analysis of 249 DEGs was performed via DAVID. The top six GO terms in the BP, CC, and MF cate- gories are shown in Figure 2. The upregulated DEGs were mainly enriched in cell division among the BP categories, the nucleus among the CC categories, and protein binding among the MF categories. The downregulated DEGs were mainly associated with transcription, DNA-templated among the BP categories, nucleus among the CC categories, and metal ion binding among the MF categories. The KEGG pathway analysis revealed that the upregulated DEGs were significantly enriched in the cell cycle, while the downregulated DEGs were mainly enriched in pathways in cancer (Figure 3).

3.3. Analysis of PPI and Module. Among 249 DEGs, 197 DEGs were assembled into a DEG PPI network complex comprising 2551 edges and 197 nodes, of which 65 were downregulated and 132 were upregulated (Figure 4(a)). The 


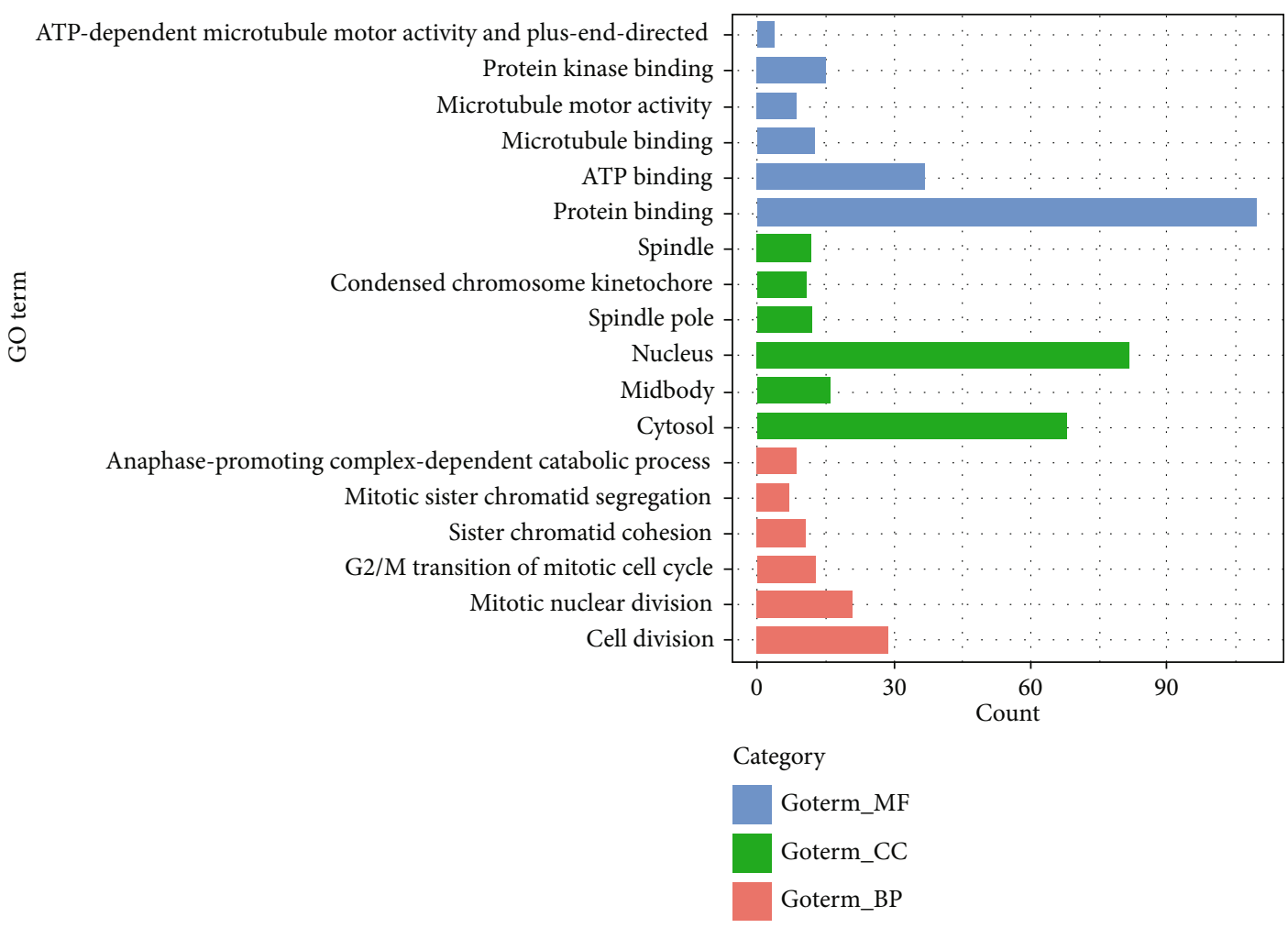

(a)

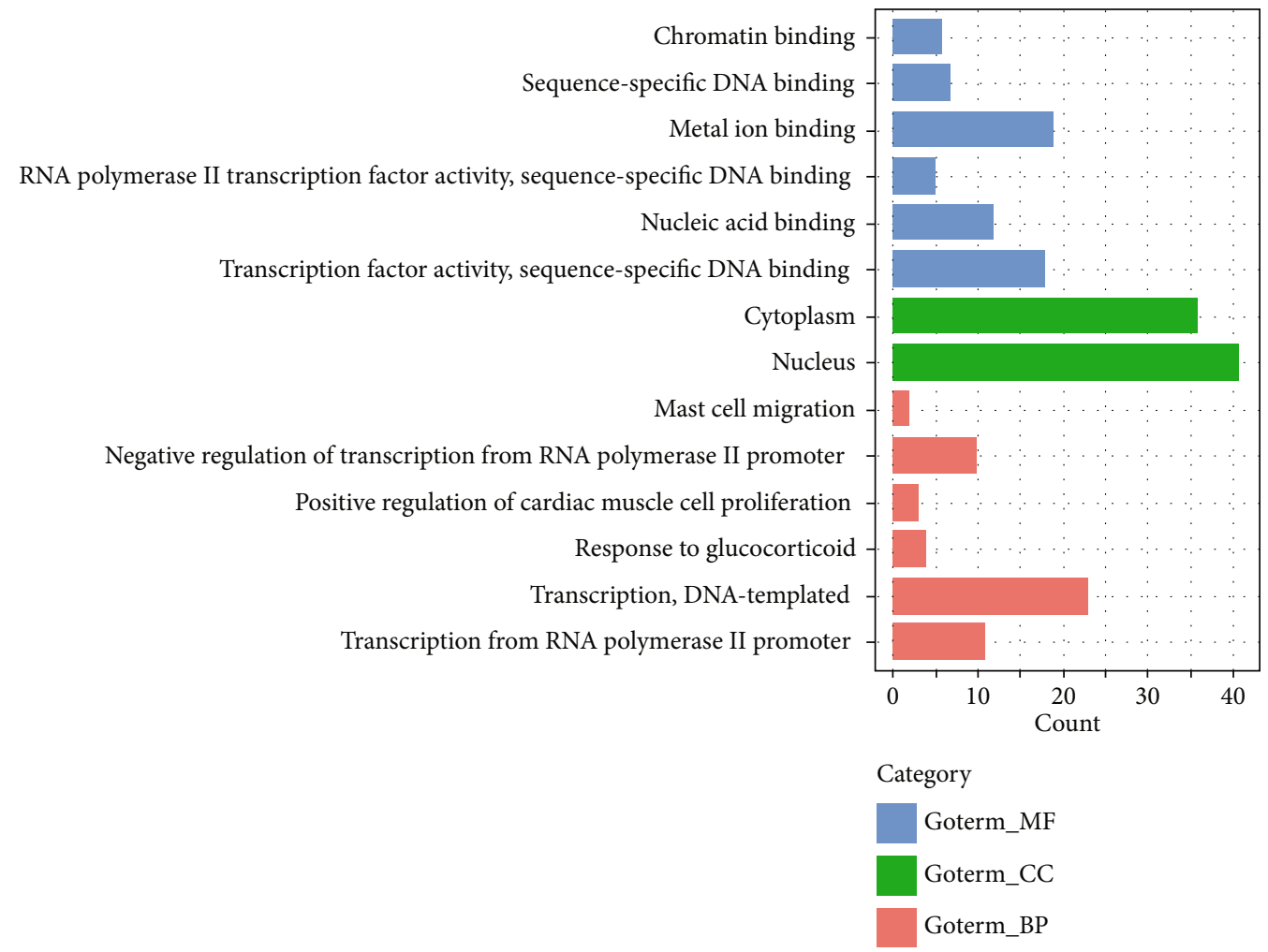

(b)

FIGURE 2: The GO term analysis of the 249 DEGs: (a) upregulated gene enrichment in GO; (b) downregulated gene enrichment in GO. GO: Gene Ontology; DEGs: differentially expressed genes. 


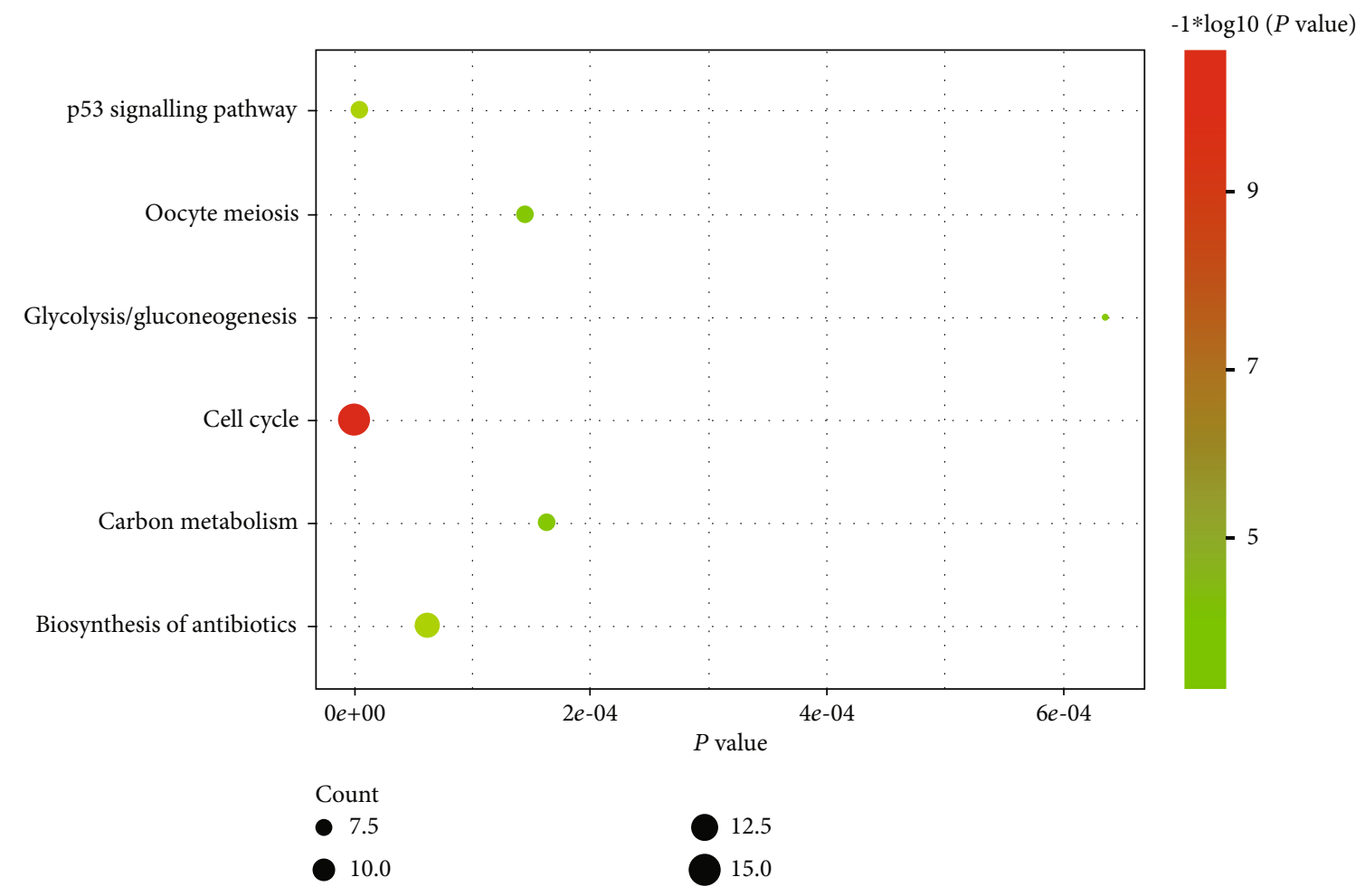

(a)

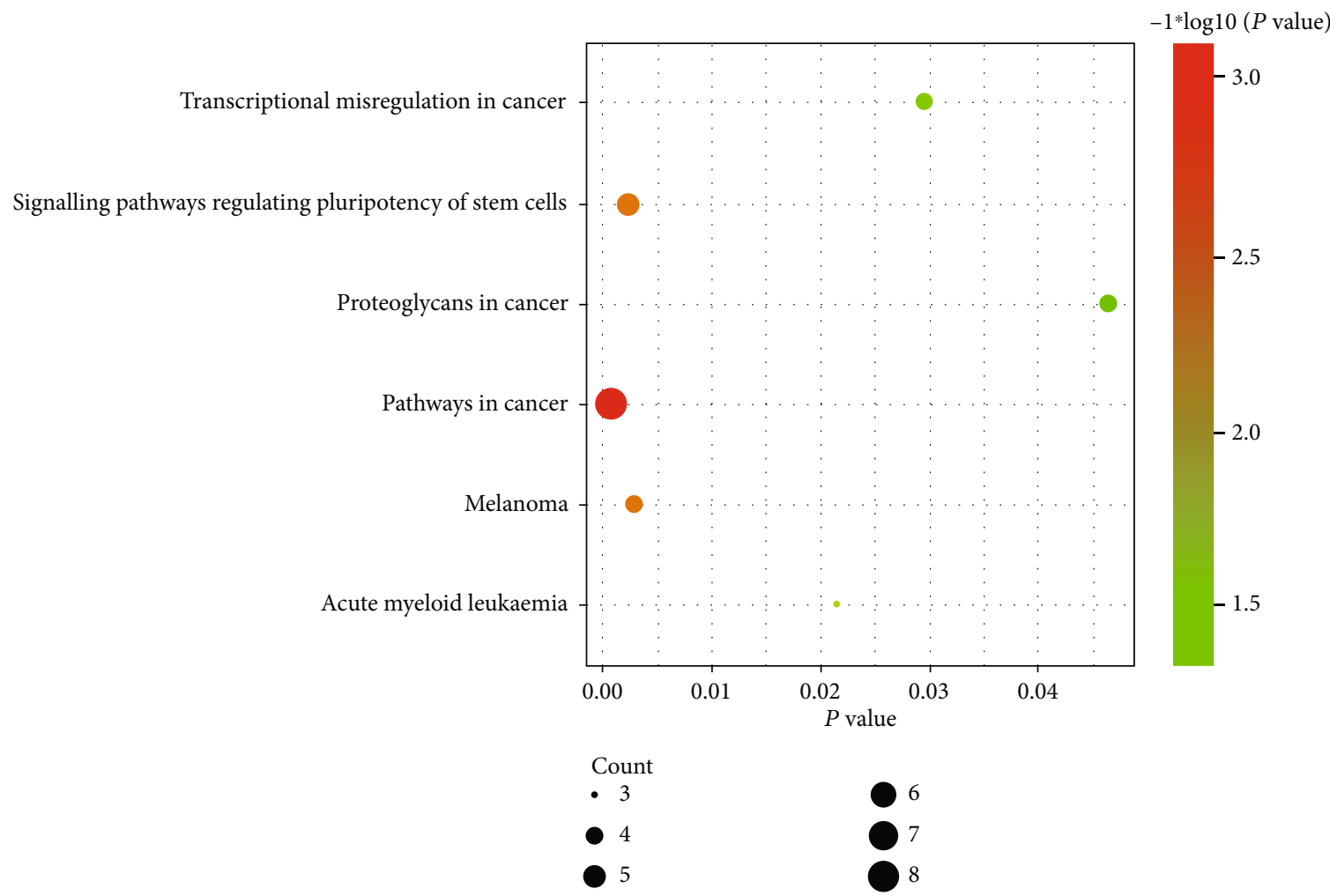

(b)

Figure 3: The KEGG pathway analysis of the 249 DEGs. (a) Upregulated genes enrichment in KEGG pathway. (b) Downregulated genes enrichment in KEGG pathway. KEGG, Kyoto Encyclopedia of Genes and Genomes; DEGs, differentially expressed genes. 


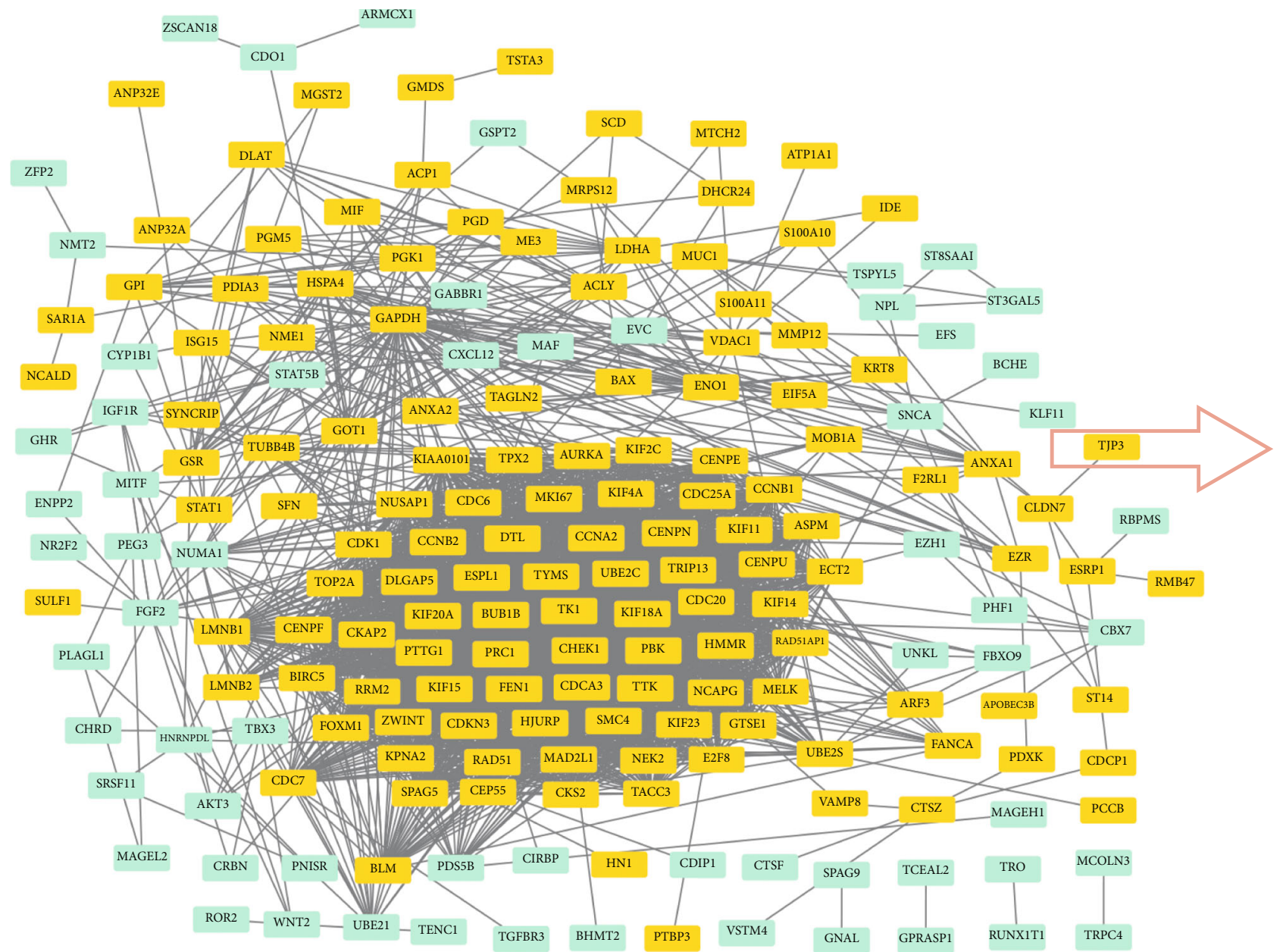

(a)

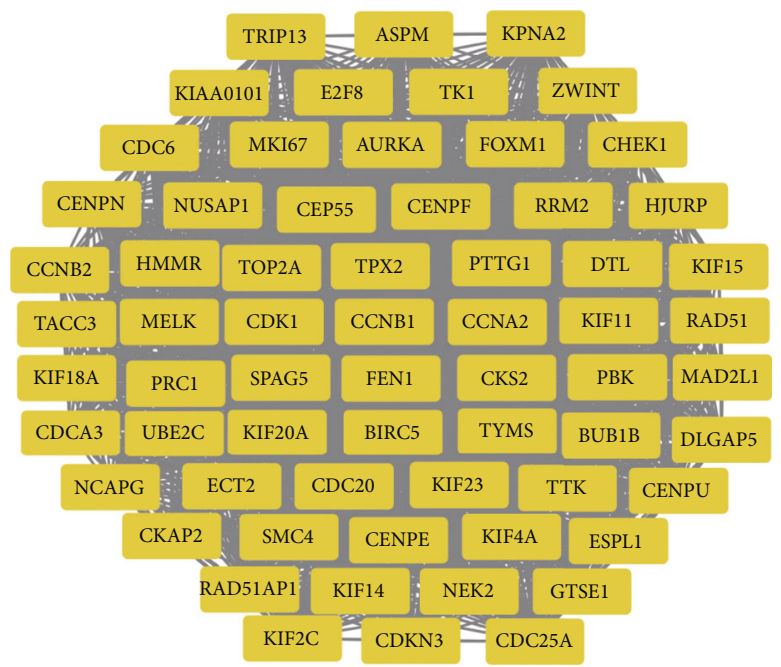

(b)

FIgURE 4: STRING and module analysis-built DEG PPI network. (a) The DEG PPI network complex had a total of 197 DEGs. The edges mean the interaction between proteins, the nodes mean proteins, and upregulated DEGs are represented by yellow rectangles and downregulated DEGs are represented by green rectangles. (b) Module analysis through the Cytoscape app ( $k$-core $=2$, max.depth $=100$, node score cutoff $=0.2$, and degree cut-off $=2$ ). 

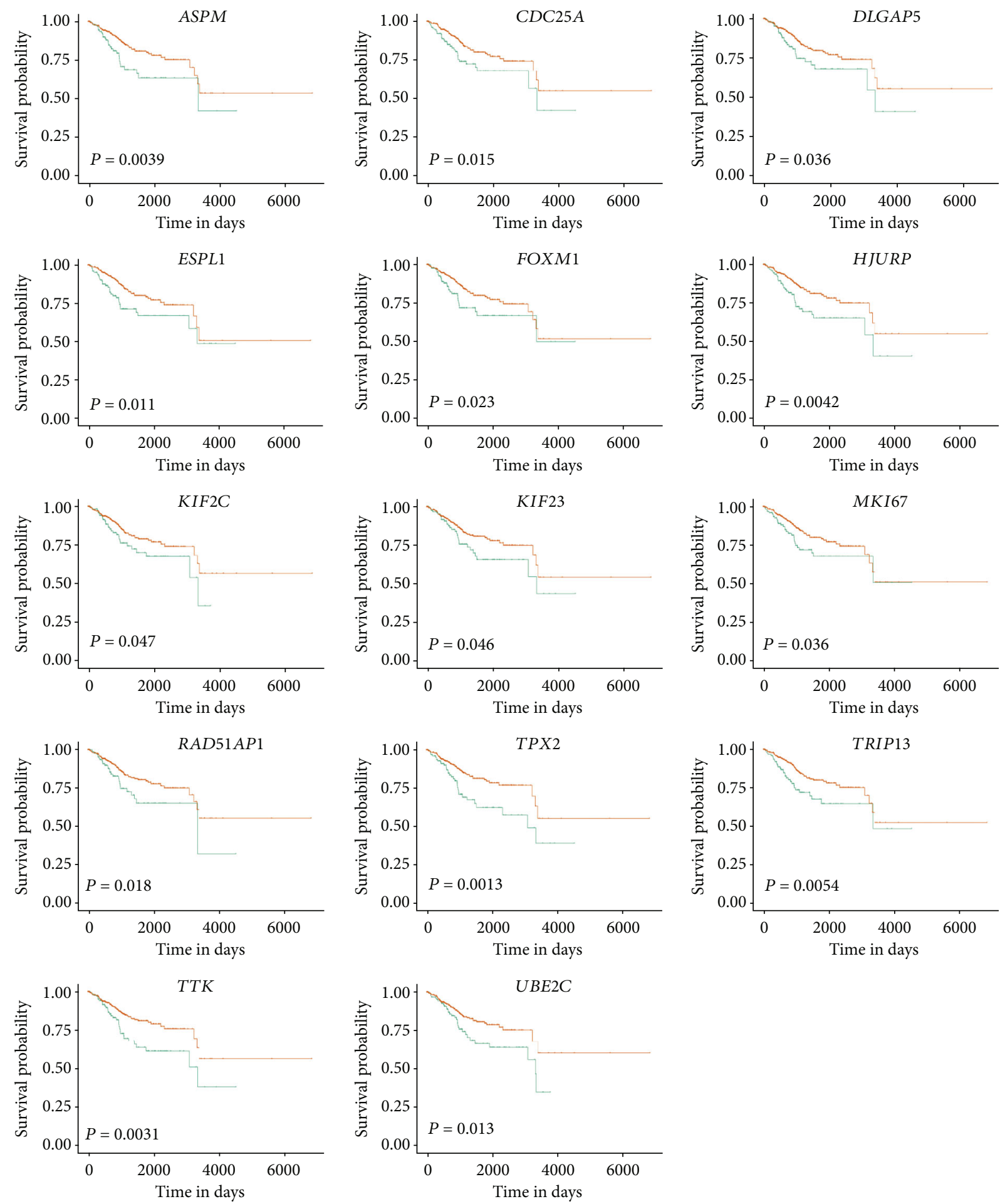

$$
\begin{aligned}
& \text { High }(n=136) \\
& \text { Low }(n=407)
\end{aligned}
$$

FIGURE 5: The Kaplan-Meier plots of the 64 core genes. Using the UALCAN online tool to verify the survival curves of the 64 core genes, and the survival rate of 14 of 64 genes was significantly poor $(P<0.05)$. 

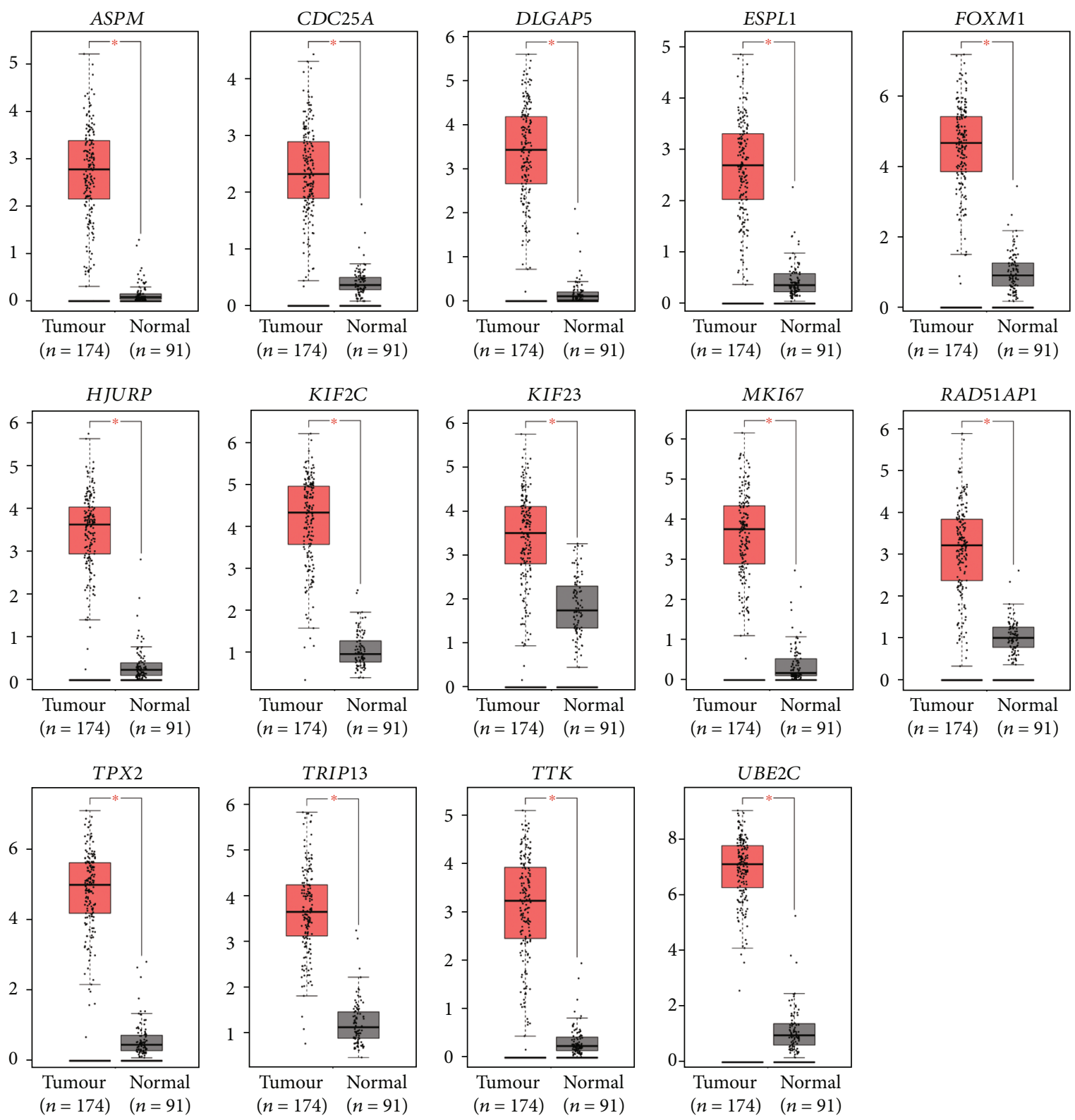

FIgURE 6: The expression of 14 genes was significantly high in endometrial cancer. The GEPIA website was applied to analyse 14 genes that were associated with poor prognosis. All of 14 genes highly expressed in endometrial cancer samples contrasted to normal samples $\left({ }^{*} P<0.01\right.$ ). Tumour tissues represented by red colour and normal tissues represented by grey colour.

DEG PPI network excluded a total of 52 DEGs (Figure 4(a)). Next, further analysis using Cytotype MCODE revealed that 64 central nodes of the 197 nodes were all upregulated genes (Figure 4(b)).

3.4. Analysis of Core Genes via UALCAN and GEPIA. UALCAN was applied to analyse the survival data for 64 core genes. The results showed that 14 genes (TRIP13, MKI67, UBE2C, RAD51AP1, DLGAP5, TTK, KIF23, TPX2, ESPL1, FOXM1, HJURP, KIF2C, CDC25A, and ASPM) were associated with significantly poorer survival, whereas 50 genes showed no significant correlations $(P<0.05$, Figure 5$)$. Next, we used GEPIA to examine the gene expression level of the 14 genes between normal endometrial specimens and EC specimens. The results indicated that all 14 genes were overexpressed in EC samples compared with normal endometrial samples $(P<0.01$, Figure 6).

3.5. KEGG Reanalysis of 14 Designated Genes by DAVID. Reanalysis of the KEGG pathway data was performed using DAVID to understand the potential biological functions of these 14 designated DEGs $(P<0.05)$. We found that three genes (TTK, CDC25A, and ESPL1) were meaningfully enriched in the cell cycle pathway $(P=0.0019$, Figure 7$)$.

3.6. Correlation Analysis between TTK, CDC25A, and ESPL1 Expression and Clinical Features. We analysed cancer genomic datasets from the UCSC Xena website and found that 


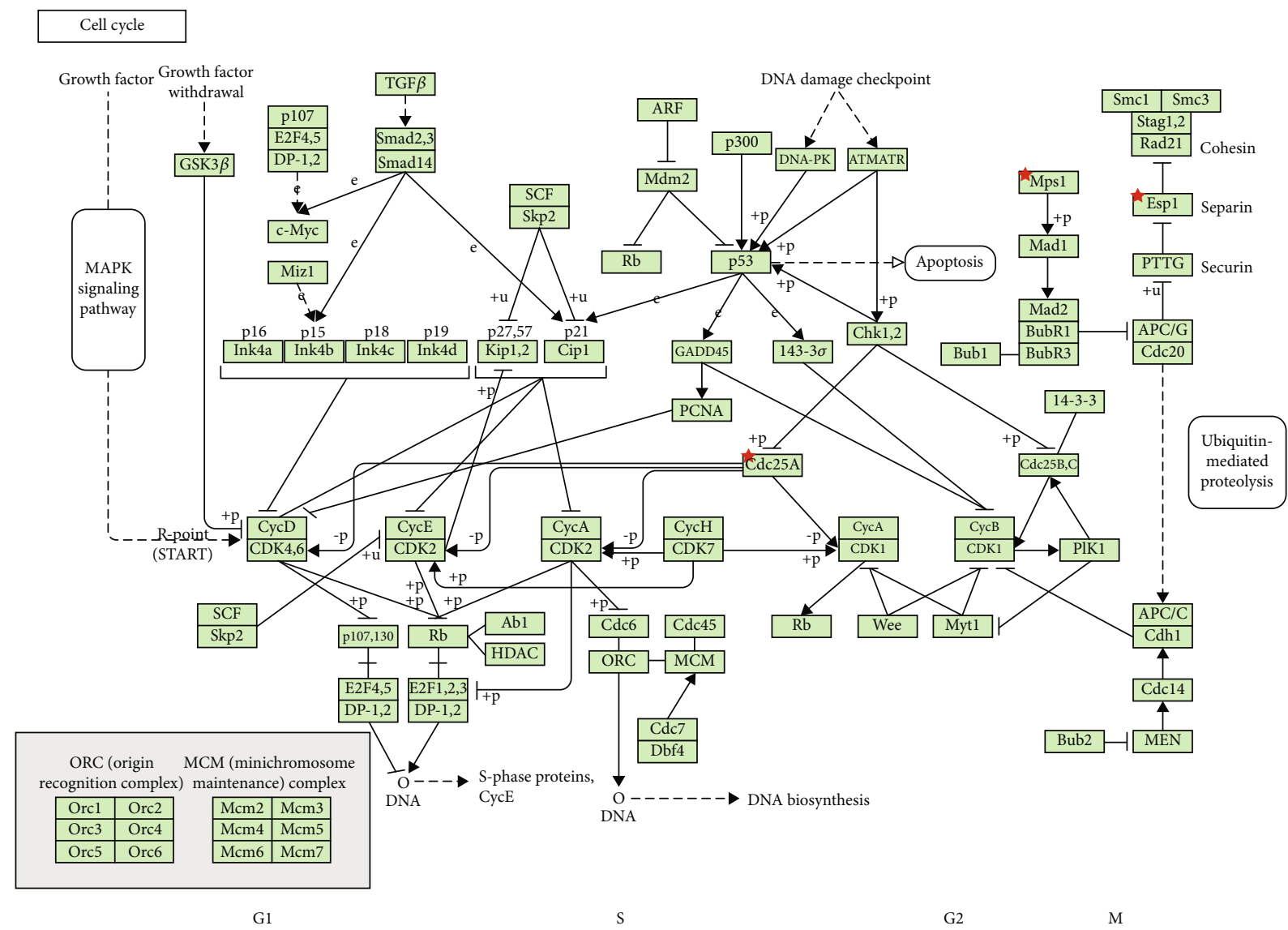

FIGURE 7: Reanalysis of 14 designated genes via KEGG pathway enrichment. Three genes (TTK, CDC25A, and ESPL1) were markedly concentrated in the cell cycle pathway. Eps1 means ESPL1. Cdc25A means CDC25A. Mps1 means TTK.

TTK, CDC25A, and ESPL1 were highly expressed in EC tissues compared with normal tissues. In addition, TTK, $C D C 25 A$, and ESPL1 showed higher expression in cancers with late stage and higher tumour grade, compared with early stage and lower tumour grade $(P<0.05$, Figures $8(\mathrm{a})$ and $8(\mathrm{~b}))$. Clinical datasets and cancer genomic datasets from the cBioPortal website were analysed, and the results showed that in cases with TTK, CDC25A, and ESPL1 amplification, EC patients had worse survival outcomes (Figure 8(c)).

\section{Discussion}

The present research performed bioinformatics analysis of two profile datasets (GSE17025 and GSE63678) to identify more useful prognostic biomarkers in EC. This study included 17 normal endometrial tissues and $98 \mathrm{EC}$ tissues. A total of 249 common DEGs (adjusted $P$ value $<0.05$ and $\left.\left|\log _{2} \mathrm{FC}\right|>1\right)$, of which 102 were downregulated $\left(\log _{2} \mathrm{FC}<-1\right)$ and 147 were upregulated $\left(\log _{2} \mathrm{FC}>1\right)$, were found through GEO2R and the Draw Venn Diagram. Next, DAVID was applied to analyse GO and KEGG pathway enrichment. The results showed that (1) among the BP terms, upregulated DEGs were mainly enriched in cell division, mitotic nuclear division, G2/M transition of mitotic cell cycle, sister chromatid cohesion, mitotic sister chromatid segregation, and anaphase-promoting complex-dependent catabolic process and downregulated DEGs were enriched in the regulation of transcription from RNA polymerase II promoter, transcription, DNA-templated, response to glucocorticoid, positive regulation of cardiac muscle cell proliferation, negative regulation of transcription from RNA polymerase II promoter, and mast cell migration; (2) among the CC terms, upregulated DEGs were markedly enriched in cytosol, midbody, nucleus, spindle pole, condensed chromosome kinetochore, and spindle and downregulated DEGs were enriched in the nucleus and cytoplasm; (3) among the MF terms, upregulated DEGs were enriched in protein binding, ATP binding, microtubule binding, microtubule motor activity, protein kinase binding, ATP-dependent microtubule motor activity, and plus-end-directed and downregulated DEGs were enriched in transcription factor activity, sequence-specific DNA binding, nucleic acid binding, RNA polymerase II transcription factor activity, sequence-specific DNA binding, metal ion binding, sequence-specific DNA binding, and chromatin binding. In the KEGG pathway analysis, upregulated DEGs were significantly enriched in cell cycle, p53 signalling pathway, biosynthesis of antibiotics, oocyte meiosis, carbon metabolism, and glycolysis/gluconeogenesis, while downregulated DEGs were enriched in pathways in cancer, signalling pathways regulating pluripotency 

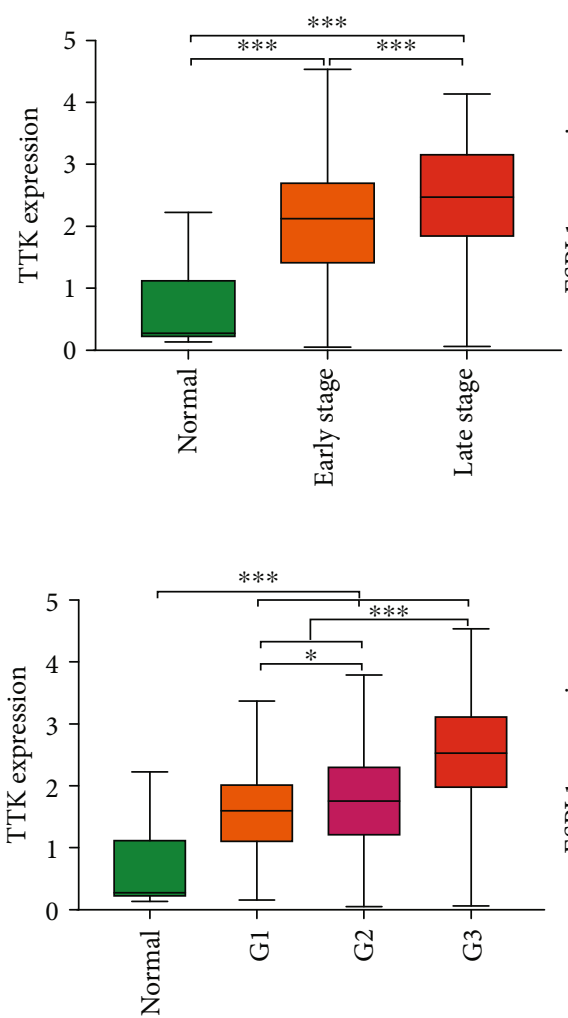

TTK

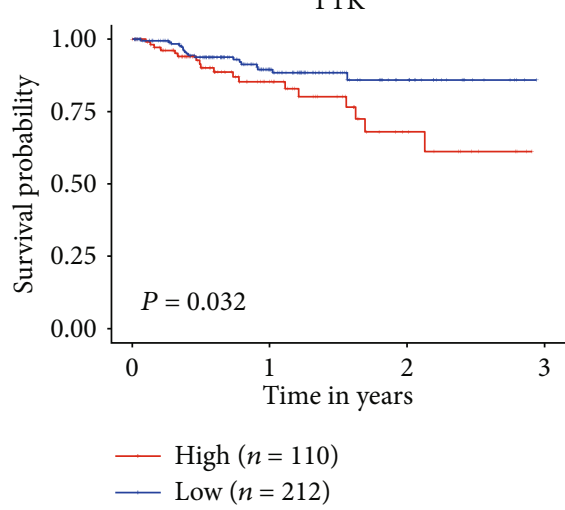

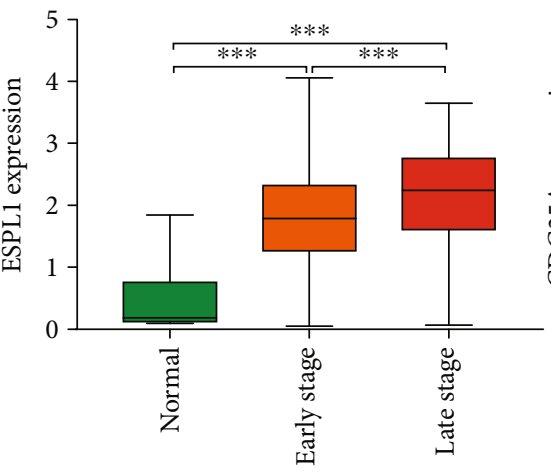

(a)
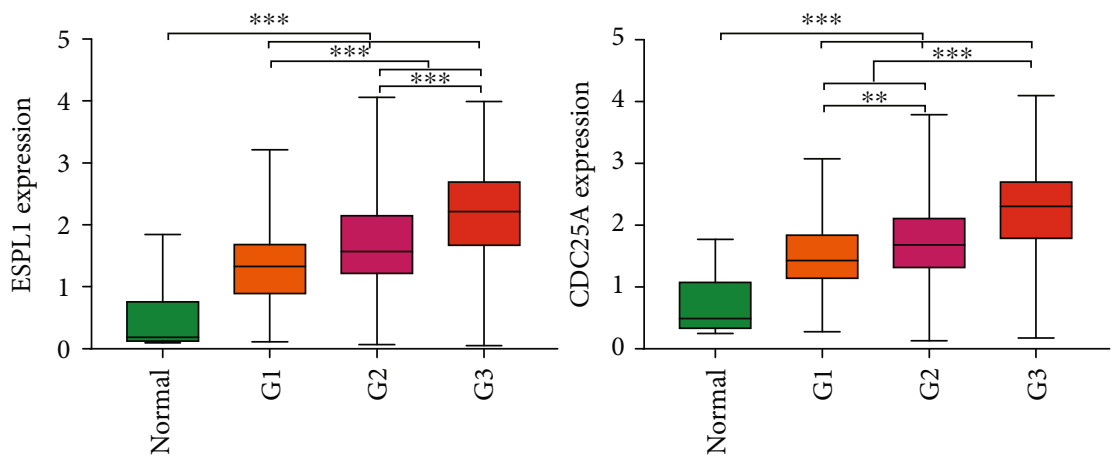

(b)
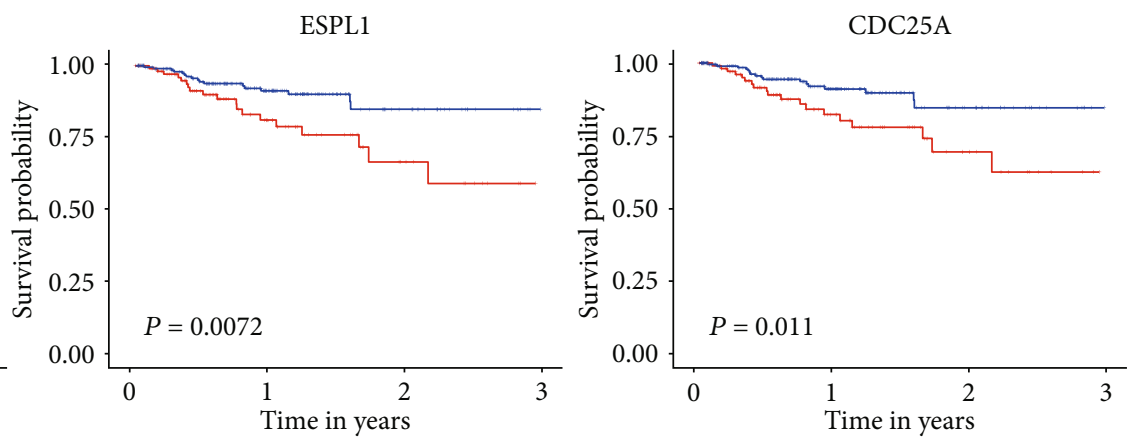

(c)

FIGURE 8: Analysis between TTK, CDC25A, and ESPL1 expression and clinical features of EC. (a) Correlation analysis between TTK, CDC25A, and ESPL1 expression with EC stage; early stage means stage I and stage II; late stage means stage III and stage IV. (b) Correlation analysis between TTK, CDC25A, and ESPL1 expression with tumour grade. (c) Correlation between TTK, CDC25A, and ESPL1 expression and survival of EC.

of stem cells, melanoma, acute myeloid leukaemia, transcriptional misregulation in cancer, and proteoglycans in cancer $(P<0.05)$. Then, we constructed a DEG PPI network complex of 2551 edges and 197 nodes by using STRING and the Cytoscape app. Next, through Cytotype MCODE analysis, 64 crucial upregulated DEGs were selected from the PPI network complex. Furthermore, 14 of 64 genes exhibited associations with significantly worse survival via UALCAN analysis. GEPIA analysis, which was used to validate these 14 genes, showed that all of these genes were more highly expressed in EC samples compared with normal endometrial samples $(P<0.01)$. DAVID was applied to reanalyse the KEGG pathway enrichment of these 14 genes. The enrichment of three genes (TTK, CDC25A, and ESPL1) in the cell cycle pathway was significant $(P<0.05)$. Lastly, TTK, CDC25A, and ESPL1 showed higher expression in cancers with late stage and higher tumour grade, which indicates that they may be potential targets for improving EC patient prognosis.

$T T K$, also known as monopolar spindle1 (Mps1), was discovered during a screening of spindle pole body replication genes in 1991 and is a kinetochore-localized protein kinase 
with dual specificity $[22,23]$. TTK is essential for mitotic checkpoints and participates in cell survival and the repair of oxidative DNA lesions. High TTK expression may help tumours overcome the challenges of an oxidative microenvironment $[24,25]$. Many studies have shown that TTK is overexpressed in several human malignant tumours, including malignant melanoma (MM), non-small cell lung carcinoma (NSCLC), prostate cancer, breast cancer, and colon cancer. Its expression is associated with the poor patient outcome. Knockdown of TTK or treatment with a TTK inhibitor could suppress tumour growth by inhibiting cell proliferation, migration, and tumorigenesis. Thus, TTK may be a potential therapeutic target for cancers [26-30].

Cell division cycle 25 (CDC25) is a bispecific phosphatase that removes phosphate groups from phosphorylated serine (Ser, S), threonine (Thr, T), and tyrosine (Tyr, Y) residues of its substrate proteins [31]. As a member of the CDC25 phosphatase family, $C D C 25 A$ is essential for the progression of the cell cycle from G1 to $S$ phase. CDC25A is involved in a variety of biological processes, such as G1/S transition, cell division, regulation of cyclin-dependent protein serine/threonine kinase activity, cell proliferation, regulation of cell cycle, DNA replication, and cellular response to UV $[32,33]$. Considered an oncogene, CDC25A is highly expressed in many types of human malignancies, such as breast cancer, ovarian cancer, head and neck cancer, colon cancer, and cutaneous squamous cell carcinoma [34-38]. Moreover, CDC25A expression was significantly associated with tumour invasion and poor tumour differentiation [39].

ESPL1 (extra spindle pole-like 1) is an endopeptidase and cysteine protease. ESPL1 is activated and cleaves the cohesin subunit RAD21 to release a sister chromatid cohesion required for chromosomal disjunction at the onset of anaphase [40-42]. ESPL1 is introduced to mitotic chromosomes to dissolve the cohesion of the sister chromatid in a DNAdependent manner, playing an important part in the progression of the cell cycle, ensuring faithful genetic inheritance $[43,44]$. Several studies have shown that ESPL1, as a candidate oncogene, is overexpressed in several types of breast cancers [45-47]. It is also a marker for mitotic activity and prognosis in breast cancers [48].

As discussed above, several studies have suggested that these three genes are associated with the progression of many types of cancer. However, our search of the PubMed database reveals that very few studies have investigated the roles of these three genes in EC. Thus, this study can provide helpful information and direction for future studies of EC.

\section{Conclusions}

In summary, we identified three DEGs (TTK, CDC25A, and ESPL1) between normal endometrial tissues and EC tissues in our bioinformatics analysis based on two mRNA expression datasets. These three genes might play important roles in the development of EC. A series of future experiments will be needed to validate these predictions. Nevertheless, these data can provide helpful information and direction for the elucidation of potential biomarkers and the biological mechanisms of EC.

\section{Data Availability}

The data used to support the findings of this study are included within the article.

\section{Conflicts of Interest}

The authors have no conflicts of interests.

\section{Acknowledgments}

The present study was funded by the Shanghai Hospital Development Center (grant no. SHDC12019113).

\section{References}

[1] P. Morice, A. Leary, C. Creutzberg, N. Abu-Rustum, and E. Darai, "Endometrial cancer," The Lancet, vol. 387, no. 10023, pp. 1094-1108, 2016.

[2] J. Lortet-Tieulent, J. Ferlay, F. Bray, and A. Jemal, "International patterns and trends in endometrial cancer incidence, 1978-2013," Journal of the National Cancer Institute, vol. 110, no. 4, pp. 354-361, 2018.

[3] R. L. Siegel, K. D. Miller, and A. Jemal, "Cancer statistics, 2019," CA: a Cancer Journal for Clinicians, vol. 69, no. 1, pp. 7-34, 2018.

[4] R. L. Siegel, K. D. Miller, and A. Jemal, "Cancer statistics, 2016," CA: a Cancer Journal for Clinicians, vol. 66, no. 1, pp. 7-30, 2016.

[5] J. V. Bokhman, "Two Pathogenetic types of endometrial carcinoma," Gynecologic Oncology, vol. 15, no. 1, pp. 10-17, 1983.

[6] A. Q. Tran and P. Gehrig, "Recent advances in endometrial cancer," F1000Research, vol. 6, p. 81, 2017.

[7] S. N. Lewin, T. J. Herzog, N. I. B. Medel et al., "Comparative performance of the 2009 International Federation of Gynecology and Obstetrics' staging system for uterine corpus cancer," Obstetrics and Gynecology, vol. 116, no. 5, pp. 1141-1149, 2010.

[8] T. Barrett, T. O. Suzek, D. B. Troup et al., "NCBI GEO: mining millions of expression profiles-database and tools," Nucleic Acids Research, vol. 33, pp. D562-D5D6, 2004.

[9] K. I. Pappa, A. Polyzos, J. Jacob-Hirsch et al., "Profiling of discrete gynecological cancers reveals novel transcriptional modules and common features shared by other cancer types and embryonic stem cells," PLoS One, vol. 10, no. 11, article e0142229, 2015.

[10] R. S. Day, K. K. McDade, U. R. Chandran et al., "Identifier mapping performance for integrating transcriptomics and proteomics experimental results," BMC Bioinformatics, vol. 12, no. 1, 2011.

[11] M. Ashburner, et al.C. A. Ball, J. A. Blake et al., "Gene ontology: tool for the unification of biology. The Gene Ontology Consortium," Nature Genetics, vol. 25, no. 1, pp. 25-29, 2000.

[12] M. Kanehisa and S. Goto, "KEGG: Kyoto Encyclopedia of Genes and Genomes," Nucleic Acids Research, vol. 28, no. 1, pp. 27-30, 2000.

[13] D. W. Huang, B. T. Sherman, and R. A. Lempicki, "Systematic and integrative analysis of large gene lists using DAVID bioinformatics resources," Nature Protocols, vol. 4, no. 1, pp. 44-57, 2009. 
[14] G. Dennis, B. T. Sherman, D. A. Hosack et al., "DAVID: Database for Annotation, Visualization, and Integrated Discovery," Genome Biology, vol. 4, no. 5, p. P3, 2003.

[15] D. Szklarczyk, A. Franceschini, S. Wyder et al., "STRING v10: protein-protein interaction networks, integrated over the tree of life," Nucleic Acids Research, vol. 43, no. D1, pp. D447-D452, 2015.

[16] P. Shannon, A. Markiel, O. Ozier et al., "Cytoscape: a software environment for integrated models of biomolecular interaction networks," Genome Research, vol. 13, no. 11, pp. 24982504, 2003.

[17] D. S. Chandrashekar, B. Bashel, S. A. H. Balasubramanya et al., "UALCAN: a portal for facilitating tumor subgroup gene expression and survival analyses," Neoplasia, vol. 19, no. 8, pp. 649-658, 2017.

[18] Z. Tang, C. Li, B. Kang, G. Gao, C. Li, and Z. Zhang, "GEPIA: a web server for cancer and normal gene expression profiling and interactive analyses," Nucleic Acids Research, vol. 45, no. W1, pp. W98-W102, 2017.

[19] E. Cerami, J. Gao, U. Dogrusoz et al., "The cBio cancer genomics portal: an open platform for exploring multidimensional cancer genomics data," Cancer Discovery, vol. 2, no. 5, pp. 401404, 2012.

[20] J. Gao, B. A. Aksoy, U. Dogrusoz et al., "Integrative analysis of complex cancer genomics and clinical profiles using the cBioPortal," Science Signaling, vol. 6, no. 269, p. pl1, 2013.

[21] D. A. Levine and The Cancer Genome Atlas Research Network, "Integrated genomic characterization of endometrial carcinoma," Nature, vol. 497, no. 7447, pp. 67-73, 2013.

[22] E. Lauzé, B. Stoelcker, F. C. Luca, E. Weiss, A. R. Schutz, and M. Winey, "Yeast spindle pole body duplication gene MPS1 encodes an essential dual specificity protein kinase," The EMBO Journal, vol. 14, no. 8, pp. 1655-1663, 1995.

[23] M. Winey, L. Goetsch, P. Baum, and B. Byers, "MPS1 and MPS2: novel yeast genes defining distinct steps of spindle pole body duplication," The Journal of Cell Biology, vol. 114, no. 4, pp. 745-754, 1991.

[24] Z. C. Yu, Y. F. Huang, and S. Y. Shieh, "Requirement for human Mps1/TTK in oxidative DNA damage repair and cell survival through MDM2 phosphorylation," Nucleic Acids Research, vol. 44, no. 3, pp. 1133-1150, 2016.

[25] H. Yang, F. Zhang, C. J. Huang et al., "Mps1 regulates spindle morphology through MCRS1 to promote chromosome alignment," Molecular Biology of the Cell, vol. 30, no. 9, pp. 10601068, 2019.

[26] A. Szymiczek, M. Carbone, S. Pastorino et al., "Inhibition of the spindle assembly checkpoint kinase Mps-1 as a novel therapeutic strategy in malignant mesothelioma," Oncogene, vol. 36, no. 46, pp. 6501-6507, 2017.

[27] S. Chen, J. Wang, L. Wang et al., "Silencing TTK expression inhibits the proliferation and progression of prostate cancer," Experimental Cell Research, vol. 385, no. 1, p. 111669, 2019.

[28] X. Chen, C. Yu, J. Gao et al., "A novel USP9X substrate TTK contributes to tumorigenesis in non-small-cell lung cancer," Theranostics, vol. 8, no. 9, pp. 2348-2360, 2018.

[29] L. Zhang, B. Jiang, N. Zhu et al., "Mitotic checkpoint kinase Mps1/TTK predicts prognosis of colon cancer patients and regulates tumor proliferation and differentiation via PKC $\alpha /$ ERK1/2 and PI3K/Akt pathway," Medical Oncology, vol. 37, no. 1, 2020.

[30] A. R. R. Maia, J. de Man, U. Boon et al., "Inhibition of the spindle assembly checkpoint kinase TTK enhances the efficacy of docetaxel in a triple-negative breast cancer model," Annals of Oncology, vol. 26, no. 10, pp. 2180-2192, 2015.

[31] J. Rudolph, "Inhibiting transient protein-protein interactions: lessons from the Cdc25 protein tyrosine phosphatases," Nature Reviews Cancer, vol. 7, no. 3, pp. 202-211, 2007.

[32] H. Sadeghi, M. Golalipour, A. Yamchi, T. Farazmandfar, and M. Shahbazi, "CDC25A pathway toward tumorigenesis: molecular targets of CDC25A in cell-cycle regulation," Journal of Cellular Biochemistry, vol. 120, no. 3, pp. 2919-2928, 2018.

[33] R. Boutros, C. Dozier, and B. Ducommun, "The when and wheres of CDC25 phosphatases," Current Opinion in Cell Biology, vol. 18, no. 2, pp. 185-191, 2006.

[34] R. Boutros, V. Lobjois, and B. Ducommun, "CDC25 phosphatases in cancer cells: key players? Good targets?," Nature Reviews Cancer, vol. 7, no. 7, pp. 495-507, 2007.

[35] H. Qin and W. Liu, "MicroRNA-99a-5p suppresses breast cancer progression and cell-cycle pathway through downregulating CDC25A," Journal of Cellular Physiology, vol. 234, no. 4, pp. 3526-3537, 2019.

[36] Y. Sun, S. Li, L. Yang et al., "CDC25A facilitates chemoresistance in ovarian cancer multicellular spheroids by promoting E-cadherin expression and arresting cell cycles," Journal of Cancer, vol. 10, no. 13, pp. 2874-2884, 2019.

[37] K. Biswas, S. Philip, A. Yadav et al., "BRE/BRCC45 regulates CDC25A stability by recruiting USP7 in response to DNA damage," Nature Communications, vol. 9, no. 1, p. $537,2018$.

[38] J. Al-Matouq, T. Holmes, B. Hammiller et al., "Accumulation of cytoplasmic CDC25A in cutaneous squamous cell carcinoma leads to a dependency on CDC25A for cancer cell survival and tumor growth," Cancer Letters, vol. 410, pp. 41-49, 2017.

[39] L. Singh, N. Pushker, S. Sen et al., "Expression of CDC25A and CDC25B phosphatase proteins in human retinoblastoma and its correlation with clinicopathological parameters," The British Journal of Ophthalmology, vol. 99, no. 4, pp. 457-463, 2015.

[40] K. Nasmyth and C. H. Haering, "Cohesin: its roles and mechanisms," Annual Review of Genetics, vol. 43, no. 1, pp. 525558, 2009.

[41] Y. Sun, M. Kucej, H. Y. Fan, H. Yu, Q. Y. Sun, and H. Zou, "Separase is recruited to mitotic chromosomes to dissolve sister chromatid cohesion in a DNA-dependent manner," Cell, vol. 137, no. 1, pp. 123-132, 2009.

[42] N. Shindo, K. Kumada, and T. Hirota, "Separase sensor reveals dual roles for separase coordinating cohesin cleavage and cdk1 inhibition," Developmental Cell, vol. 23, no. 1, pp. 112-123, 2012.

[43] M.-F. B. Tsou, W.-J. Wang, K. A. George, K. Uryu, T. Stearns, and P. V. Jallepalli, "Polo kinase and separase regulate the mitotic licensing of centriole duplication in human cells," Developmental Cell, vol. 17, no. 3, pp. 344-354, 2009.

[44] M. Papi, E. Berdougo, C. L. Randall, S. Ganguly, and P. V. Jallepalli, "Multiple roles for separase auto-cleavage during the G2/M transition," Nature Cell Biology, vol. 7, no. 10, pp. 1029-1035, 2005.

[45] P. Finetti, A. Guille, J. Adelaide, D. Birnbaum, M. Chaffanet, and F. Bertucci, "ESPL1 is a candidate oncogene of luminal B breast cancers," Breast Cancer Research and Treatment, vol. 147, no. 1, pp. 51-59, 2014.

[46] N. Zhang and D. Pati, "Biology and insights into the role of cohesin protease separase in human malignancies," Biological 
Reviews of the Cambridge Philosophical Society, vol. 92, no. 4, pp. 2070-2083, 2017.

[47] N. Zhang, G. Ge, R. Meyer et al., "Overexpression of separase induces aneuploidy and mammary tumorigenesis," Proceedings of the National Academy of Sciences of the United States of America, vol. 105, no. 35, pp. 13033-13038, 2008.

[48] N. Gurvits, E. Loyttyniemi, M. Nykanen, T. Kuopio, P. Kronqvist, and K. Talvinen, "Separase is a marker for prognosis and mitotic activity in breast cancer," British Journal of Cancer, vol. 117, no. 9, pp. 1383-1391, 2017. 